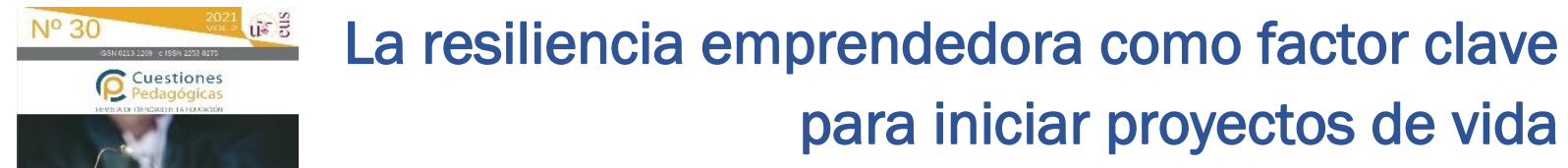

Entrepreneurial resilience as a key factor in initiating life

projects

\title{
Elisabet Montoro-Fernández
}

Universidad Loyola

emontoro@uloyola.es

https://orcid.org/0000-0003-1775-5568

\author{
M. Camino Escolar-Llamazares \\ Universidad de Burgos \\ cescolar@ubu.es \\ https://orcid.org/0000-0003-2448-0267
}

Resumen: Actualmente, existe una gran preocupación por difundir una cultura emprendedora en todos los países de la Unión Europea. Esta inquietud por contribuir al desarrollo económico ha llevado a apostar por la educación emprendedora, incorporándola en todos los niveles del sistema educativo. El emprendimiento se ha convertido, así pues, en un fenómeno completamente afianzado en España, donde se ha puesto especial interés en promover su desarrollo. Esto ha llevado a abordar el concepto del emprendimiento desde diferentes ámbitos en los últimos años, pudiéndolo diferenciar desde distintas perspectivas. En nuestro caso, relacionamos el emprendimiento con la iniciativa personal, de modo que la persona pone en marcha voluntariamente cualquier tipo de acción. No obstante, para que la acción de emprender verdaderamente funcione, el individuo necesita establecer proyectos de vida que tengan significado y sentido para él, favoreciendo, así, su bienestar y desarrollo personal. Del mismo modo, necesita desarrollar la capacidad de resiliencia, es decir, la capacidad de resistir, ya que, durante el transcurso de la persecución de objetivos vitales, puede encontrarse con numerosos
Abstract: Nowadays there is a great concern to spread an entrepreneurial culture in all the European Union. This need to contribute to the economic development, has led to a commitment to entrepreneurial education, incorporating it in every level of the educational system. In this way, entrepreneurship has become a fully established phenomenon in Spain, where promoting its development has been a special interest. This has led to approach the concept of entrepreneurship from different areas in recent years, being able to differentiate it from different perspectives. In our case, we relate entrepreneurship to personal initiative, so is the person who voluntarily initiates any type of action. However, for this to truly work the person needs to establish life projects that have meaning for they, favoring their well - being and personal development. In the same way, the person needs to develop the capacity for resilience, that is, the ability to resist, since, during the course of the pursuit of vital objectives, they may encounter numerous obstacles that could make it difficult or impede the fulfillment of these. In this sense, it is essential that the person develop the capacity for entrepreneurial resilience, understood as the ability to adequately face any adversity and, at 
obstáculos de diversa índole, pudiendo dificultar o impedir el cumplimiento de estos. En este sentido, resulta fundamental que la persona desarrolle la capacidad de resiliencia emprendedora, entendida como la capacidad para afrontar adecuadamente cualquier adversidad y, al mismo tiempo, generar de nuevo proyectos más acordes a sus circunstancias personales. the same time, generate new projects more in line with their personal circumstances.

\begin{tabular}{|c|c|c|c|c|}
\hline $\begin{array}{l}\text { Palabras clave: } \\
\text { emprendimiento, } \\
\text { resiliencia. }\end{array}$ & $\begin{array}{l}\text { Capacidad, } \\
\text { desarrollo }\end{array}$ & $\begin{array}{l}\text { educación, } \\
\text { humano, }\end{array}$ & $\begin{array}{l}\text { Keywords: } \\
\text { entrepreneurship, } \\
\text { resilience. }\end{array}$ & $\begin{array}{r}\text { education } \\
\text { development }\end{array}$ \\
\hline
\end{tabular}

\section{Introducción ${ }^{1}$}

La sociedad actual presenta una necesidad de implantar e impulsar la educación desde una óptica predominantemente socioeconómica, de forma que las personas puedan adaptarse a las nuevas demandas de la economía del conocimiento, elevar la productividad, así como la competitividad, incrementar la calidad y el nivel de empleo, mejorar la empleabilidad y tener una mayor cohesión grupal (COM, 2002, 2004, 2006; Eurydice España-Redie, 2015). La primera gran crisis económica mundial de este siglo no ha hecho sino reafirmar los postulados de formación de los ciudadanos europeos a través de la competitividad, la cohesión social y la integración en la economía del conocimiento.

Todo ello ha provocado que el emprendimiento se haya convertido en un fenómeno con gran auge en los diferentes países europeos. Diversos estudios e investigaciones estudian en profundidad este fenómeno, siendo redefinido desde múltiples perspectivas (económica, social y personal, principalmente) y distintos ámbitos científicos a lo largo del tiempo (Erkkila, 2000; Bridge, 2017; Bernal y Liñán, 2018). Numerosas investigaciones corroboran que, en la actualidad, existe una falta de consenso acerca del término emprender (Toro y Ortegón, 1999; Villamor y Prieto, 2014; Peña, Cárdenas, Rodríguez y Sánchez, 2015; Láckeus, 2017).

A pesar de que no parece haber un acuerdo común sobre el fenómeno del emprendimiento, lo cierto es que la Unión Europea ha estado preocupada e interesada en fomentar acciones emprendedoras, con la intención de contribuir al desarrollo económico de cada país. Concretamente, marcó determinadas directrices

\footnotetext{
${ }^{1}$ Este artículo está vinculado al proyecto «Formación del Potencial Emprendedor. Generación de un Modelo Educativo de Identidad Emprendedora», con Referencia PID2019-104408GB-I00, dentro del Plan Estatal 2017-2020 de Proyectos I+D+i (Generación de Conocimiento), financiado por: Ministerio de Ciencia e Innovación - Agencia Estatal de Investigación/10.13039/501100011033.
} 
a través de sus Consejos y Programas, que desembocaron en la creación de una nueva legislación educativa. El Consejo de Lisboa, en el año 2000, supuso el punto de partida para fomentar la cultura emprendedora en Europa. A partir de entonces, se han ido promoviendo diferentes estrategias (Education, Audiovisual and Culture Executive Agency, 2012) para integrar la educación emprendedora en el ámbito educativo (EACEA, 2016). En el caso de España, la legislación educativa ha favorecido gradualmente la incorporación de la cultura emprendedora en la escuela (LOCE, 2002; LOE, 2006 y LOMCE, 2013), aunque con diferencias dependiendo de la etapa educativa y de las comunidades autónomas (Global Entrepreneurship Monitor, 2014).

Si bien, en un primer momento, la educación emprendedora ha estado exclusivamente relacionada con la educación empresarial, lo cierto es que un conjunto de transformaciones sociales y cambios en nuestro sistema ha influido en el campo educativo y, como resultado, la educación emprendedora ha experimentado un nuevo giro en los países europeos en estos últimos años, estando vinculada con una educación especializada en la formación y sensibilización hacia el emprendimiento, alejado de la perspectiva de crear únicamente empresas.

El emprendimiento se relaciona ahora con una serie de actitudes y capacidades, así como con la iniciativa. Esto hace referencia a lo que se ha denominado potencial emprendedor, esto es, el conjunto de capacidades y habilidades que desarrolla un individuo para configurar su identidad emprendedora (Bernal y Cárdenas, 2017). Estas capacidades aluden, inevitablemente, a la dimensión personal del sujeto, por lo que el emprendimiento es comprendido desde una perspectiva personal. Entre las capacidades que más destacan, se encuentran el sentido crítico, la creatividad, el liderazgo, la motivación de logro, la responsabilidad y la resolución de problemas (Athayde, 2012). Otras investigaciones amplían esta lista con las capacidades personales de autonomía e iniciativa (Bernal y Cárdenas, 2014; Montoro, 2021).

Bajo esta perspectiva, se han proporcionado acciones y programas encaminados a formar al alumnado en la competencia de emprender, de modo que estos pudieran adquirir una serie de actitudes y capacidades relacionadas con el espíritu empresarial y emprendedor. Más específicamente, se elaboró el documento de "Competencias clave para un aprendizaje a lo largo de la vida", donde se definen las siguientes competencias (Comisión Europea, 2014): 1) la comunicación en la lengua materna, 2) la comunicación en lenguas extranjeras, 3) la competencia matemática y las competencias básicas en ciencia y tecnología, 4) la competencia digital, 5) aprender a aprender, 6) las competencias sociales y cívicas, 7) el sentido de la iniciativa y el espíritu de empresa y 8) la conciencia y la expresión cultural.

En el mismo sentido, la Organización para la Cooperación y el Desarrollo Económico (OCDE) y la Dirección General de Educación y Cultura de la Comisión 
Europea concretan ciertas competencias claves, teniendo relevancia entre ellas la de actuar de manera autónoma y el espíritu emprendedor (DeSeCo, 2006).

Desarrollar la capacidad de autonomía implica que la persona tenga la iniciativa para construir y dirigir proyectos vitales y planes con verdadero valor y significatividad para ella. Para lograrlo, es necesario adquirir un conjunto de valores afines con la responsabilidad y disponer de un criterio propio sólido que contribuya a edificar los cimientos morales de los proyectos de vida. De igual modo, es fundamental que el individuo aprenda a crear y a gestionar estos proyectos personales mediante la planificación, implementación y evaluación. En este sentido, es conveniente promover el desarrollo de una autoestima y autoconcepto positivos que aumente la autoconfianza para mostrar un comportamiento caracterizado por la iniciativa, creatividad y empuje personal (Marina, 2010; Benítez y Carrasco, 2019).

\section{Emprender proyectos de vida}

De manera paulatina, la sociedad actual ha sufrido grandes transformaciones que han desembocado en la apertura y la pluralidad, favoreciendo un mayor abanico de posibilidades de acción para el individuo. Los márgenes de actuación se han extendido hasta tal punto, que las personas disponen de múltiples opciones de elección para promover su desarrollo personal, no pudiendo delegar en los demás la creación de su futuro, sino que deben ser ellos mismos quienes decidan qué camino escoger. La amplitud de posibilismos conlleva a un mayor espacio para la iniciativa personal, de modo que el individuo pueda desarrollar la capacidad para diseñar, planificar y llevar a cabo todos los proyectos vitales que crea adecuados para su bienestar personal.

En este sentido, el emprendimiento es entendido como iniciativa personal, es decir, como la capacidad que poseen las personas para dirigir sus vidas a través de ciertos recursos personales, teniendo como finalidad crear proyectos de vida que resulten significativos y motivantes, de forma que generen bienestar personal en el individuo (Cárdenas, 2012; Herrera y Gutiérrez, 2014; Gorostiaga, Balluerka, Ulacia, y Aliri, 2018).

La acción de emprender está supedita, por un lado, a la iniciativa personal y, por otro lado, a los proyectos de vida. Analizar de forma independiente estos componentes resulta necesario para comprender las bases del emprendimiento desde la perspectiva que aquí se plantea.

\section{Iniciativa}

Desde nuestra óptica, el emprendimiento está vinculado al concepto de iniciativa personal, lo que se ve reflejado en el desarrollo y crecimiento personal que produce la generación de proyectos de vida (Fontaines, Palomo y Velásquez, 2015). 
Lisbona y Frese (2012) planteaban que la iniciativa personal constituía "un conjunto de fenómenos que caracterizan una situación determinada" (2012, p.23). Este planteamiento supone que la iniciativa no se trata únicamente de una conducta aislada, sino que se compone por un conjunto de ellas. Siguiendo este supuesto, la iniciativa personal se compone por las siguientes características (Frese, 2001; Ulacia et al., 2017): a) conductas autoiniciadas, que consistiría en los objetivos que se autoimpone la persona en lugar de ser asignados por los demás. El individuo inicia una determinada conducta por propia voluntad, sin que nadie del entorno le incite a realizarla. No la siente como una tarea o una obligación a cumplir, sino como algo que quiere hacer. Para lograr el objetivo, la persona debe cumplir dos pasos; por una parte, desarrollar esa idea y, por otra parte, asumir la responsabilidad para llevarla a cabo. La idea no tiene por qué ser desconocida, pero sí debe ser aplicada en un contexto donde no se haya puesto en práctica antes, b) proactividad, es decir, la persona debe anticipar una determinada situación o problema específico, demostrando la capacidad de adelantarse a los acontecimientos y dificultades que puedan presentarse, de manera que pueda ofrecer diferentes alternativas para solucionarlas en la mayor brevedad posible. Para que esto funcione realmente, las conductas proactivas deben establecerse en un periodo de largo plazo para poder atender a las demandas del contexto, c) persistencia y capacidad para superar los obstáculos, la primera es considerada una cualidad positiva y necesaria para lograr determinados objetivos, ya que cuando se introducen mejoras se originan, inevitablemente, diversos cambios que no siempre funcionan al primer intento. A causa de esto, es conveniente tener la persistencia para poder superar las dificultades que pueden aparecer durante la consecución del objetivo fijado. Con la segunda, aludimos a un conjunto de herramientas emocionales para autorregular la conducta y esta pueda ser encaminada al cumplimiento del objetivo. De este modo, la persona tendría los recursos necesarios para afrontar cualquier tipo de adversidad, protegiendo sus objetivos a pesar de las dificultades. Esta característica está vinculada estrechamente con la capacidad de resiliencia, es decir, la capacidad para afrontar adecuadamente cualquier situación traumática y salir indemne de la misma.

La consecución de los objetivos puede verse interrumpida ante la existencia de problemas que provocan emociones negativas, pudiendo, incluso, bloquear los planes de acción. Por este motivo, es fundamental que la persona desarrolle la capacidad de resiliencia, de manera que pueda gestionar estas emociones, sin perder de vista la finalidad que se ha propuesto en un principio. Así pues, desarrollar esta capacidad supone cierta garantía de éxito en el cumplimiento de los objetivos establecidos.

Frese, Garst, y Fay (2007) también añadieron otro rasgo de la iniciativa, la capacidad para modificar el ambiente. Para poder realizar la iniciativa, habrá situaciones donde no haya otra alternativa que modificar el entorno. Esto no significa que siempre se deba introducir cambios contextuales, habrá que valorar la circunstancias para ver si estos son factibles o no. 
Teniendo en cuenta todo lo anterior, podemos decir que la iniciativa personal requiere la puesta en práctica de determinados componentes cognitivos, emocionales y volitivos del individuo para generar y concretar proyectos de vida. Estos componentes, a su vez, están formados por las siguientes fases: definición del objetivo, recopilación de la información y fijación del pronóstico, elaboración del plan, ejecución y evaluación del mismo, así como feedback (Aziz y Petrovich, 2019; Laguia, Jaén, Topa y Moriano, 2019).

Más concretamente, la primera fase consiste en el establecimiento del objetivo, precisando de una definición y descripción en profundidad de este, lo más exhaustivo posible, de forma que no dé lugar a errores, dudas ni malinterpretaciones. La persona debe recordar la necesidad de agenciarse el objetivo como propio, teniendo claro que no es algo autoimpuesto ni externo, sino que nace de sus deseos y necesidades personales. Asimismo, el objetivo debe ser establecido a largo plazo, para que haya tiempo de anticiparse, por un lado, a los problemas u obstáculos que se puedan presentar y, por otro lado, a las oportunidades que puedan ser aprovechadas. Es evidente que, durante el transcurso del cumplimiento del objetivo, aparecerán problemas que deberán ser superados mediante la persistencia y la constancia. Para hacer frente a estos problemas y resolverlos con éxito, será más fácil si la persona siente un sentimiento fuerte de responsabilidad sobre el objetivo y cuenta con una gran variedad de recursos que autorregulen adecuadamente los procesos emocionales negativos que pueden conllevar determinadas dificultades, protegiéndose ante ellas. La siguiente fase requiere de una planificación concienzuda y organizada que permita poner en práctica la iniciativa sin imprevistos. Se trata de elaborar un plan de acción que se ajuste al máximo al logro del objetivo. Para poder diseñarlo adecuadamente, es vital que la persona analice los recursos de los que dispone, recopilar todos los datos posibles para tener la seguridad de cuáles acciones son las más factibles y cuáles no, así como valorar las diferentes oportunidades y los problemas que probablemente surgirán. El desarrollo del plan posiblemente sea la fase más importante, ya que supone la conexión entre el pensamiento y la acción. La viabilidad entre uno y otro es difícil, puesto que algunas veces generamos ideas innovadoras y creativas, que seguramente pensamos que tienen muchas probabilidades de éxito y, sin embargo, no se llevan a cabo. Este hecho pone en evidencia la relevancia de crear planes que sean verdaderamente realizables, especificando al detalle los pasos que se deben seguir para conseguirlos. No podemos interpretar este procedimiento como algo inflexible, sino más bien como algo pautado, reflexionado y organizado para obtener el éxito, de manera que no haya demasiado margen para improvisar. La última fase se refiere a la pertinencia de evaluar la ejecución del plan, revisando en todo momento si la planificación ha sido adecuada. Además, se debe solicitar una retroalimentación o feedback, con la intención de comprobar si el proceso ha tenido realmente éxito. La evaluación del plan es recomendable realizarla durante el mismo proceso, ya que permite modificar a tiempo todo lo que no esté dando resultado. Al mismo tiempo, es conveniente 
controlar los estados emocionales asociados a la aparición de resultados, especialmente los negativos, que son los que pueden generar frustraciones $\mathrm{y}$, finalmente, el abandono de la iniciativa.

Como hemos podido observar, el proceso de la iniciativa personal no está exento de dificultades y barreras que pueden impedir el cumplimiento de los proyectos personales. Por esta razón, resulta necesario mejorar o potenciar todos los aspectos o características de la iniciativa personal. Para ello, Lisbona y Frese (2012) proponen los siguientes elementos: autoeficacia, orientación al cambio, manejo de los errores y manejo del estrés.

La autoeficacia se genera a través de la información obtenida procedente de cuatro fuentes (Lisbona, Palacia, Salanova y Frese, 2018): 1) Logros de ejecución. En el momento en el que la persona consigue realizar una acción que le supone un gran desafío, aumenta su autoeficacia. Por tanto, la clave se encontraría en diseñar entornos y actividades que faciliten mayor posibilidad de cumplir la tarea con éxito, siendo la persona consciente de ello a través de la retroalimentación. 2) Experiencia vicaria. Cuando la persona visualiza o sospecha cómo los demás realizan determinadas acciones y proyectos exitosos, puede llegar a creer que ella misma tiene las capacidades necesarias para hacerlo también, obteniendo el mismo resultado. Esta creencia es muy beneficiosa para aquellas personas que no conocen sus propias capacidades o bien para aquellas que no tienen experiencia en ese tipo de proyecto. 3) Persuasión verbal. A través de la comunicación y, concretamente, de las palabras, podemos incrementar la confianza en otra persona, lo que supondría, a su vez, una mejora de la autoeficacia. Esto podría funcionar incluso cuando el individuo habla consigo mismo, autoconvenciéndose de que es capaz de realizar una determinada tarea. La clave parece ser que la persona perciba la acción como un reto. Hay que tener en cuenta que la tarea no puede ser excesivamente complicada de conseguir para evitar frustraciones y que la persona debe recibir rápidamente un feedback. 4) Estado fisiológico. Para poder llevar a cabo cualquier tipo de tarea es imprescindible reunir una serie de condiciones físicas.

La orientación al cambio implica que el individuo muestre cierta flexibilidad. No puede oponer resistencia a que los acontecimientos cambien. Esto puede suponer una gran dificultad, ya que las personas suelen resistirse al cambio por temor a una pérdida de control (Córica, 2020). Aunque las conductas iniciativas requieren cierto grado de control, lo cierto es que cuando alguien se obsesiona excesivamente con controlar todos los aspectos de una situación y todas sus variables, se sumerge en un estado de negatividad y ansiedad, que no ayuda en absoluto a la consecución del objetivo. También encontramos a personas que presentan una gran rigidez cognitiva, que es un aspecto de la personalidad que fomenta la resistencia al cambio. Son personas rígidas de pensamiento, que no suelen aceptar las opiniones de los otros una vez que ya han tomado una decisión. Se muestran reacios a cambiar su decisión, aun cuando tienen pruebas de que están cometiendo un error. En estos casos, es 
necesario aplicar un entrenamiento basado en la obligación de realizar modificaciones para combatir esa rigidez. Sánchez, Maggi y Paredes (2019) consideran que otra explicación para la resistencia al cambio puede ser el miedo al fracaso. Un entrenamiento para esto sería obligar al individuo a experimentar fracasos, de modo que logre perder el miedo a cometer errores.

Aprender de los errores y saber gestionarlos puede convertirse en una tarea sumamente difícil. Una forma de entrenar esto podría ser planificar la manera de prevenirlos o anticiparse cuando estos sean inevitables. Así, la persona podría introducir cambios en el proceso para solucionar las dificultades que se presenten. Los errores están íntimamente relacionados con los estados emocionales a los que inducen (González, 2019). Así pues, para poder manejarlos adecuadamente, es necesario dirigir los esfuerzos a minimizar, por una parte, el miedo a equivocarse y, por otra, la reacción emocional negativa que eso genera. Es importante tener presente que la persona debe sentirse segura, ya que, en ocasiones, la inseguridad provoca que se oculten los errores por miedo a dañar la autoestima.

Cuando los estados emocionales negativos no se gestionan, aparece el estrés, conduciendo en muchos casos a estados altos de ansiedad y depresión que desembocan en cambios fisiológicos (Puigbó, Edo, Rovira, Limonero y Fernández, 2019). En el momento en el que la persona se siente amenazada por el entorno y cree que no tiene herramientas para hacerle frente, se produce estrés. Cuando decide iniciar conductas puede experimentar altos niveles de estrés y evitarlo supone no desarrollar esas conductas. Además, en muchas ocasiones, las personas adoptan estrategias de afrontamiento que no son útiles para gestionar el estrés. Un estilo de afrontamiento válido es el coping, que se encarga de gestionar la emoción negativa provocada por dicho estrés (Gustems, Calderón y Calderón, 2019). Este estilo de afrontamiento surge cuando ya se ha dado la situación estresante, por lo que es cierto que no serviría para anticipar los problemas ni prevenirlos. Para esto último, existen estrategias activas de afrontamiento que pueden permitir a las personas prevenir las dificultades antes de que ocurran, pudiendo solucionarlas en el momento. Sin embargo, en otras ocasiones, no es posible prever los problemas, por lo que es necesario disponer de estrategias que permitan gestionar el estrés provocado por situaciones presentes. Es, por tanto, en estos casos, donde el coping se desmarca como la estrategia idónea para regular el estrés y resolver las problemáticas derivadas de este.

\section{Proyectos de vida}

Al igual que ocurre con el caso de la iniciativa, el otro pilar sobre el que se edifica el emprendimiento es el proyecto de vida. Para poner en marcha acciones de emprendimiento es necesario que las personas posean objetivos y proyectos claros que determinen la dirección de sus acciones. Estos proyectos dan sentido a la trayectoria personal, así como permiten la aparición y desarrollo de las estrategias 
precisas y adecuadas que favorecen el cumplimiento de los objetivos deseados (Mejía, 2019).

Todo el mundo necesita tener un propósito en la vida (purpose in life en inglés), un motor que les haga seguir adelante cada día y con el que se sientan plenamente realizados. Que las personas tengan un propósito en la vida responde a una fuerza primaria (Frankl, 1991).

Ryff (1989) señala que tener ese propósito significa establecer metas personales y dirigir las acciones de la persona hacia la consecución de las mismas, de modo que le genere bienestar personal. Además, sólo así su pasado y su presente adquiere sentido y significado.

En la ejecución de los proyectos de vida, la iniciativa personal juega un papel clave, puesto que, si la persona asume la responsabilidad de desarrollar proyectos vitales, significaría que tiene un grado adecuado de iniciativa personal.

Los proyectos personales son "secuencias de acciones interrelacionadas con la finalidad de conseguir algunas metas personales" (Palys y Little, 1983, p. 1222). Responden a una serie de inquietudes y curiosidades que la persona tiene a lo largo de su vida (Little, 1983). Crear proyectos de vida implica que el individuo presenta una orientación optimista dirigida hacia el futuro. No obstante, no podemos olvidar que la realización de estos proyectos no depende exclusivamente de su esfuerzo, sino que están influenciados por todas las variables del contexto en el que se encuentra sumergido. Todo esto hace necesario la evaluación de los proyectos, analizando los recursos personales y contextuales y todo lo que puede influir en su implementación para comprobar si son viables y realizables.

El desarrollo de proyectos vitales requiere de una estructuración de metas que ayude a configurar una red de hitos. La persona debe asegurarse de seguir cada hito para poder llevar a cabo el proyecto. Es imprescindible que defina y establezca diferentes proyectos que aumenten su bienestar personal, pues sólo así podrá adquirir un compromiso real. Centrarse en proyectos que no les resulte lo suficientemente satisfactorio conduce, con mayor probabilidad, al fracaso y al abandono. Por lo tanto, las metas deben tener un sentido real y una gran significatividad para que la persona se sienta motivada y desee fervientemente realizarlas, a pesar de todos los problemas que puedan ir apareciendo durante el proceso. Al margen de la estructuración de metas, será necesario implementar también estrategias eficaces para lograr los propósitos establecidos (Mejía, 2019). Sólo de esta manera, la persona sentirá una gran motivación y encaminará su conducta hacia el objetivo deseado.

Para alcanzar los propósitos fijados, existen dos tipos de metas (Ortony, Clore y Collins, 1996): las instrumentales, que sirven para conseguir otras metas y las de 
consecución, compuestas por las metas que pueden ser logradas. Podríamos decir que la utilidad de las metas instrumentales es cumplir las metas de consecución. Estas últimas serían los proyectos de vida establecidos previamente, convirtiéndose en aquello que la persona desea.

Con base en lo anterior, podemos decir que las personas encuentran el significado y la satisfacción en sus vidas cuando utilizan las metas y planes como herramientas para ello (Chan, Domínguez y Santos, 2020). El individuo establece proyectos de vida y metas personales, sabiendo de antemano la finalidad que quiere alcanzar, mediante la elaboración de planes de acción y empleando diferentes estrategias para tener éxito. Así, consigue obtener los resultados deseados. En suma, el esfuerzo que emplea para lograr sus proyectos vitales contribuye de manera significativa a su bienestar psicológico y, por tanto, a su crecimiento personal (Reyes, Martínez y Ponce, 2017; Freire, Del Mar, Núñez y Valles, 2017).

En definitiva, es de gran relevancia que las personas tengan metas vitales que otorguen sentido a sus vidas y cuyo cumplimiento les hagan sentirse realizadas a todos los niveles.

\section{Conclusiones}

A pesar de que existe una gran preocupación por promover una cultura emprendedora en todos los ámbitos y, especialmente, en el contexto educativo, lo cierto es que apenas hay investigaciones que contemplen la perspectiva personal del emprendimiento asociado a la iniciativa y a los proyectos de vida. Sería conveniente profundizar en las motivaciones de la persona, en todo aquello que para ella tenga sentido e implique un verdadero compromiso, garantizando la acción de emprender en proyectos significativos, ya que, sin un motivo real por el que vivir, hacer frente a todos los obstáculos y seguir adelante, las personas no emprenderían ningún tipo de proyecto.

Del mismo modo, deberíamos contemplar el diseño de acciones y programas que fomentaran el desarrollo de la capacidad de resiliencia para afrontar adecuadamente cualquier tipo de adversidad en el transcurso del cumplimiento de los proyectos de vida, garantizando, una vez más, la culminación de estos.

En conclusión, para emprender es necesario desarrollar la capacidad de resiliencia emprendedora, que constituiría el esfuerzo personal y la voluntad para derrotar cualquier adversidad y obstáculo que se presente, además de poseer la habilidad de formar planes vitales y proyectos personales que garanticen el éxito en la vida de los individuos (Montoro, 2021). Esta capacidad sería una pieza fundamental para iniciar proyectos de vida significativos y resistir y superar las dificultades que se puedan encontrar hasta lograr hacerlos realidad. 
Desarrollar la capacidad de resiliencia emprendedora significaría dotar a las personas de recursos y herramientas que aumenten sus posibilidades de realización humana (Bernal, 2003). Estos recursos personales contribuirían a implementar con éxito los proyectos de vida, permitiendo a la persona la capacidad de conducirse a sí misma a través de la vida.

\section{Referencias}

Athayde, R. (2012). The impact of enterprise education on attitudes to enterprise in young people: an evaluation study. Education +Training, 54(8/9), 709-726.

Aziz, C. y Petrovich, F. (2019). La teoría de la acción, en acción. Nota Técnica. Líderes educativos, Centro de Liderazgo para la Mejora Escolar.

Benítez, E. y Carrasco, R. (2019). Aproximación a las características emprendedoras del gestor universitario. Arandu-UTIC. Revista Científica Internacional de la Universidad Tecnológica Intercontinental, 6(1), 145-162.

Bernal, A. (2003). El constructo madurez personal como competencia y sus posibilismos pedagógicos. Revista Española de Pedagogía, 225, 243-261.

Bernal, A. y Cárdenas, A. (2014). La formación de emprendedores en la escuela y su repercusión en el ámbito personal. Una investigación narrativa centrada en el Programa EME. Revista Española de Pedagogía, 72(257), 124-143.

Bernal, A. y Cárdenas, A. (2017). Evaluación del potencial emprendedor en escolares. Una investigación longitudinal. Educación XXI, 20(2), 73-94.

Bernal, A. y Liñán, F. (2018). The personal dimensión of an entrepreneurial competence: an aprroach from the Spanish basic education context. En A. Fayolle (ed.), A Research Agenda for Entrepreneurship Education (pp.262280). Edwatd Elgar Publishing.

Bolívar, A, y Pereira, M. (2006). El Proyecto DeSeCo sobre la definición y selección de competencias clave. Introducción a la edición Española.

Bridge, S. (2017). Is "entrepreneurship" the problem in entrepreneurship education? Education + Training, 59(7/8), 740-750.

Cárdenas, A. (2012). Trayectorias formativas y la dimensión emprendedora de la identidad personal (Tesis doctoral, Universidad de Sevilla).

Chan, I., Domínguez, N. y Santo, D. (2020). Sentido de vida y establecimiento de metas en estudiantes de bachillerato. ACADEMO. Revista de Investigación en Ciencias Sociales y Humanidades, 7(1), 22-32.

Comisión de las Comunidades Europeas (2004). Plan de Acción: El programa europeo a favor del espíritu empresarial. Bruselas.

Comisión de las Comunidades Europeas (2006). Fomentar la mentalidad empresarial. Bruselas.

Comisión Europea (2002a). Entrepreneurship. Flash Eurobarometer 134. Directorate General Enterprises. 
Comisión Europea (2014). Informe final del grupo de expertos. Proyecto del procedimiento best sobre educación y formación en el espíritu empresarial. Dirección General de Empresa.

Consejo Europeo (2000). Consejo Europeo de Lisboa 23 y 24 de marzo de 2000. Conclusiones de la Presidencia.

http://www.europarl.europa.eu/summits/lis1 es.htm.

Córica, J. (2020). Resistencia docente al cambio: Caracterización y estrategias para un problema no resuelto. RIED. Revista Iberoamericana de Educación a Distancia, 23(2), 255-272.

EACEA (2016). Entrepreneurship in Europe Eurydice Report Education at School. Luxembourg: Publications Office of the European Union.

Erkkilä, K. (2000). Entrepreneurial Education. Mapping the debates in the United States, the United Kingdom and Finland. Garland Publishing.

Eurydice España-Redie (2015). La educación para el emprendimiento en el sistema educativo español. Año 2015. Ministerio de educación, cultura y deporte. España. https://sede.educacion.gob.es/publiventa/d/20842/19/0

Fontaines, T., Palomo, M. y Velásquez, M. (2015). Resiliencia como Componente de la Actitud Emprendedora de los Jóvenes Universitarios. Revista Educación y Desarrollo Social. 9(1), 160-183.

Frankl, V. (1991). El hombre en busca de sentido (12ª ed.). Herder.

Freire, C., Del Mar, M., Núñez, J. y Valle, A. (2017). Estructura factorial de las Escalas de Bienestar Psicológico de Ryff en estudiantes universitarios. European Journal of Education and Psychology, 10(1), 1-8.

Frese, M. (2001). Personal initiative (PI): The theoretical concept and empirical findings. En M. Erez, U. Kleinbeck y H. Thierry (eds.), Work motivation in the context of a global economy (pp. 99-110). Erlbaum.

Frese, M., Garst, H. y Fay, D. (2007). Making things happen: Reciprocal relationships between work characteristics and personal initiative in a four-wave longitudinal structural equation model. Journal of Applied Psychology, 92(4), 1084-1102.

Global Entrepreneurship Monitor (2014). Informe GEM España 2014. Santander: Universidad de Cantabria.

González, D. (2019). El acierto de las equivocaciones. Aportaciones de la neurociencia cognitiva al proceso de aprendizaje. PULSO. Revista de Educación, (42), 167-180.

Gorostiaga, A., Balluerka, N., Ulacia, I. y Aliri, J. (2018). Evaluación de la iniciativa personal en el ámbito educativo y su relación con la actitud emprendedora y el rendimiento académico. Revista Iberoamericana de Diagnóstico y Evaluacióne Avaliação Psicológica, 3(48), 105-116.

Gustems, J., Calderón, C. y Calderón, D. (2019). Stress, coping strategies and academic achievement in teacher education students. European Journal of Teacher Education, 42(3), 375-390. 
Herrera, K. y Gutiérrez, J.M. (2014). El emprendimiento como iniciativa para creación de empresas: análisis de la perspectiva psicológica y contextual. Revista de Ciencias Sociales, (2), 288-302.

Láckeus, M. (2017). Does entrepreneurial education trigger more or les neoliberalism in education? Education+Training, 59(6), 635-650.

Laguia, A., Jaén, I., Topa, G. y Moriano, J. (2019). University environment and entrepreneurial intention: the mediating role of the components of the theory of planned behaviour. Revista de Psicología Social, 34(1), 137-167.

Ley Orgánica 10/2002, de 23 de diciembre, de Calidad de la Educación. Boletín Oficial del Estado (BOE), 307, 24 de diciembre de 2002.

Ley Orgánica 2/2006, de 3 de mayo, de Educación. Boletín Oficial del Estado (BOE), 106, mayo.

Ley Orgánica 8/2013, de 9 de diciembre, para la Mejora de la Calidad Educativa. Boletín Oficial del Estado (BOE), 295, diciembre.

Lisbona, A. y Frese, M. (2012). Iniciativa personal. Ediciones Pirámide.

Lisbona, A., Palaci, F., Salanova, M. y Frese, M. (2018). The effects of work engagement and self-efficacy on personal initiative and perfomance. Psicothema, 30(1), 89-96.

Little, B. (1983). Personal projects: A rationale and method for investigation. Environment and Behavior, 15(3), 273-309.

Marina. J.A. (2010). La competencia de emprender. Revista de Educación, 351, 4971.

Márquez, E. (2019). Educación superior, ¿motor de cambio o factor de exclusión social? Universitaria, 2(11), 16-17.

Mejía, P. (2019). Educación: Orientación Vocacional y Profesional, garantía de derechos y construcción de proyectos de vida. Revista Ciencia UNEMI, 12(30), 87-102.

Montoro, E. (2021). Resiliencia emprendedora y crecimiento personal: construcción de un modelo para la educación inclusiva (Tesis Doctoral, Universidad de Sevilla).

Ortony, A., Clore, G. y Collins, A. (1996). La estructura cognitiva de las emociones. Siglo XXI de España Editores S.A.

Palys, T. y Little, B. (1983). Perceived life satisfaction and the organizations of personal project systems. Journal of Personality and Social Psychology, 44(6), 1221-1230.

Peña, J., Cárdenas, A., Rodríguez, A. y Sánchez, E. (2015). La cultura emprendedora como objetivo educativo: marco general y estado de la cuestión. En L. Núñez (coord.) Cultura emprendedora y Educación (19-60). Universidad de Sevilla.

Puigbó, J., Edo, S., Rovira, T., Limonero, J. y Fernández, J. (2019). Influencia de la inteligencia emocional percibida en el afrontamiento del estrés cotidiano. Ansiedad y estrés, 25(1), 1-6. 
Reyes, A., Martínez, M. y Ponce, M. (2017). Bienestar psicológico, metas y rendimiento académico. Vertientes Revista Especializada en Ciencias de la Salud, 19(1), 29-34.

Ryff, C. (1989). Beyond Ponce de Leon and life satisfaction: new directions in quest of successful aging. International Journal of Behavioral Development, 12(1), 35-55.

Sánchez, M., Maggi, M. y Paredes, M. (2019). Resistencia al cambio en las organizaciones: propuesta para minimizarlo. Palermo Business Review, (19), 39-53.

Toro, J. y Ortegón, A. (1999). Corriendo el telón del concepto emprendedor. Revista Escuela de Administración de Negocios, 37, 133-141. Bogotá: Universidad EAN.

Ulacia, I., Gorostiaga, A., Balluerka, N. y Aliri, J. (2017). Desarrollo de la iniciativa personal: impacto de una intervención aplicada al ámbito educativo. Journal for the Study of Education and Development, Infancia y Aprendizaje, 40(2), 277-301.

Villamor, P. y Prieto, M. (2014). El espíritu emprendedor en educación: un análisis pedagógico. Procedia. Social and Behavorial Sciences, 139, 153-159. 